\title{
Simulation of the ATLAS New Small Wheel (NSW) System
}

\author{
Huacheng Cai*, on behalf of the ATLAS Muon collaboration \\ University of Illinois at Urbana Champaign, Urbana, Illinois, USA \\ E-mail: huacheng.cai@cern.ch
}

\begin{abstract}
The instantaneous luminosity of the Large Hadron Collider (LHC) at CERN will be increased up to a factor of five with respect to the present design value by undergoing an extensive upgrade program over the coming decade. In order to benefit from the expected high luminosity performance that will be provided by the Phase-1 upgraded LHC, the first station of the ATLAS muon endcap Small Wheel (SW) system will need to be replaced by a New Small Wheel (NSW) detector. The NSW will have to operate in a high background radiation region, while reconstructing muon tracks with high precision as well as furnishing information for the Level-1 trigger. This document will summarize the developed simulation model and the importance of the NSW system for the improvement of the muon reconstruction efficiency and muon identification.
\end{abstract}

5th International Conference on Micro-Pattern Gas Detectors (MPGD2017)

22-26 May, 2017

Philadelphia, USA

${ }^{*}$ Speaker. 


\section{New Small Wheel upgrade for future LHC runs}

The instantaneous luminosity of the LHC is planned to be increased up to $3 \times 10^{34} \mathrm{~cm}^{-2} \mathrm{~s}^{-1}$ from 2021 (Run3), and further to $7 \times 10^{34} \mathrm{~cm}^{-2} \mathrm{~s}^{-1}$ from 2026 (High-Luminosity LHC), which is a factor of five to seven higher than the original design value. In the meanwhile, the average number of interactions per bunch crossing $(<\mu>)$ will be increased up to 80 in Run3, and up to 160 in the High-Luminosity LHC. In the high luminosity environment, the muon performance is expected to degrade due to an increase of the fake muon triggers and efficiency drop of the precision trackers. In order to solve these two problems, the inner station of the ATLAS muon end-cap Small Wheel (SW) system [1] will need to be replaced by the New Small Wheel (NSW) [3]. The NSW is going to be installed in the ATLAS detector in the forward region of $1.3<|\eta|<2.7$ during the second long LHC shutdown (2019 to 2021).

Compared to the current SW system, the NSW will provide additional information to the muon Level-1 trigger in the end-cap region, in order to reduce fake triggers arising from particles that are not high $p_{T}$ muons originating at the interaction point (IP). During Run1, the end-cap muon triggers were formed by the Big Wheel (BW) TGC only. Therefore one of the main sources of the fake triggers was particles generated in the material between SW and BW, as shown in case B of Figure 1. Also it was hard to reject the particles which were not generated from IP, but crossed the $\mathrm{BW}$ at an angle similar to that of real high $p_{T}$ muons, as shown case $\mathrm{C}$ of Figure 1 . Therefore, requesting the hit coincidence between the NSW and BW can effectively reduce the fake triggers and maintain a high efficiency in the high rate environment.

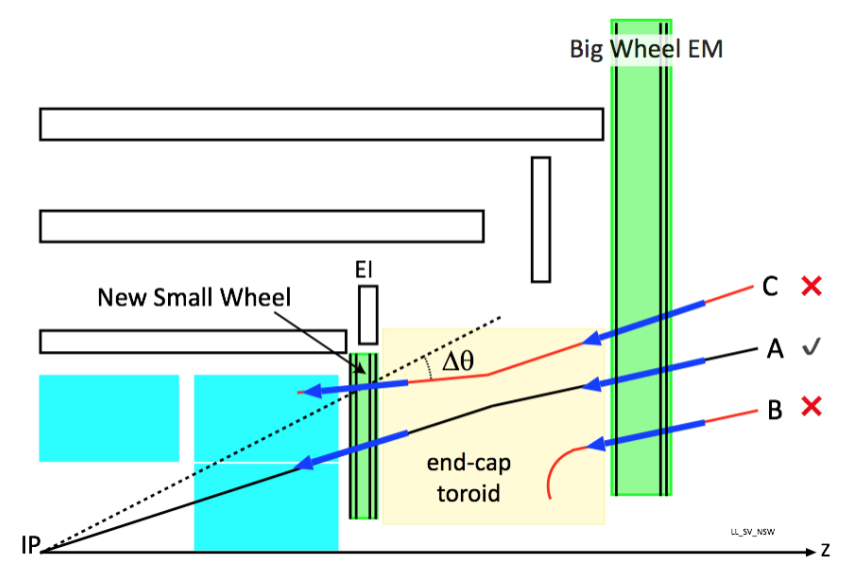

Figure 1: Schematic of the muon end-cap trigger [2]. At Run1, the end-cap trigger decision was produced by only the BW. It included a lot of fake triggers because the BW triggers accept B and $\mathrm{C}$ tracks as well as A tracks. This problem can be handled by the NSW that would reject B and C tracks.

Two new technologies will be employed in the NSW: large-area resistive Micromegas (MM) and small-strip Thin Gap Chamber (sTGC). The sketches of the layout of a sTGC and an MM detector are shown in Figure 2. Each sector of NSW is composed by 8 sTGC layers and 8 MM layers, where the MM layers are sandwiched between the sTGC layers. Both technologies will be 
used for triggering and tracking independently. This document will focus on a detailed studies of the MM detector in the NSW, including the final design and validation of the readout electronics path (Section 2), the algorithm for MM trigger (Section 3), and a study of the MM performance using a detailed Monte Carlo (MC) simulation (Section 4).
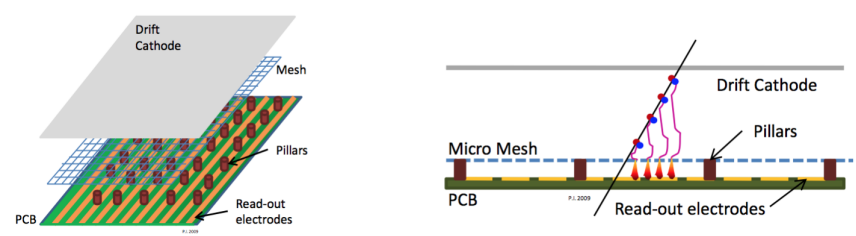

(a) Sketch of MM detector

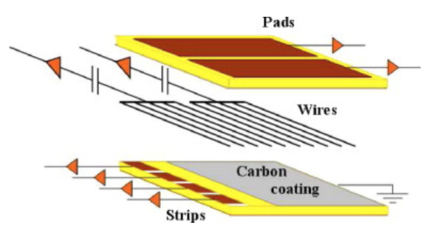

(b) Sketch of sTGC detector

Figure 2: Two new technologies implemented in the NSW: large-area resistive Micromegas (MM) and small-strip Thin Gap Chamber (sTGC) [3].

\section{The NSW MM trigger data flow path}

A schematic view of the NSW MM DAQ data flow is shown in Figure 3. The MM strip signals are sent to the 64-channel VMM ASIC. The Address-in-Real-Time (ART) from each 32 front-end VMMs (four MM front end cards, MMFE-8) is serially transmitted, point-to-point, to a companion digital ASIC on the ART Data Driver Card (ADDC). Then the ART data with the corresponding bunch crossing identification is sent to the trigger processor (in USA15 cavern) via optical links.

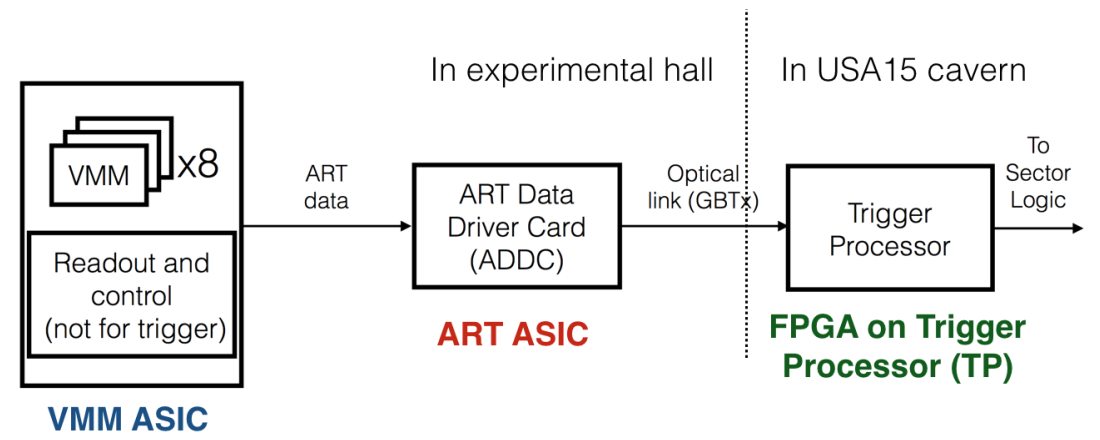

Figure 3: A schematic view of the NSW MM DAQ data flow.

There are a total of 1024 ADDCs, which means the total number of channels is more than two million. It is therefore extremely challenging to process all the channels in the Level-1 trigger latency [4], and a data suppression technique is needed. Each VMM will only output the strip address of the first channel read out of 64 channels. This is because the first read out always corresponds to the shortest drift distance. In addition, a first strip in time is a good approximation for hit position due to fine strip pitch, which is about $0.4 \mathrm{~mm}$. 
Figure 4 shows the timing distribution of the ART signal in a VMM ART simulation with single high $p_{T}$ muon events. A small charge threshold is used in this simulation in order to get the optimum results of the timing threshold. As can be seen, a 25 (50) ns time window has an efficiency of 87 (99)\%, which corresponds to the hit efficiency of the ART signal. This implies that the $50 \mathrm{~ns}$ time window will be promising to get a high efficiency for the trigger processor.

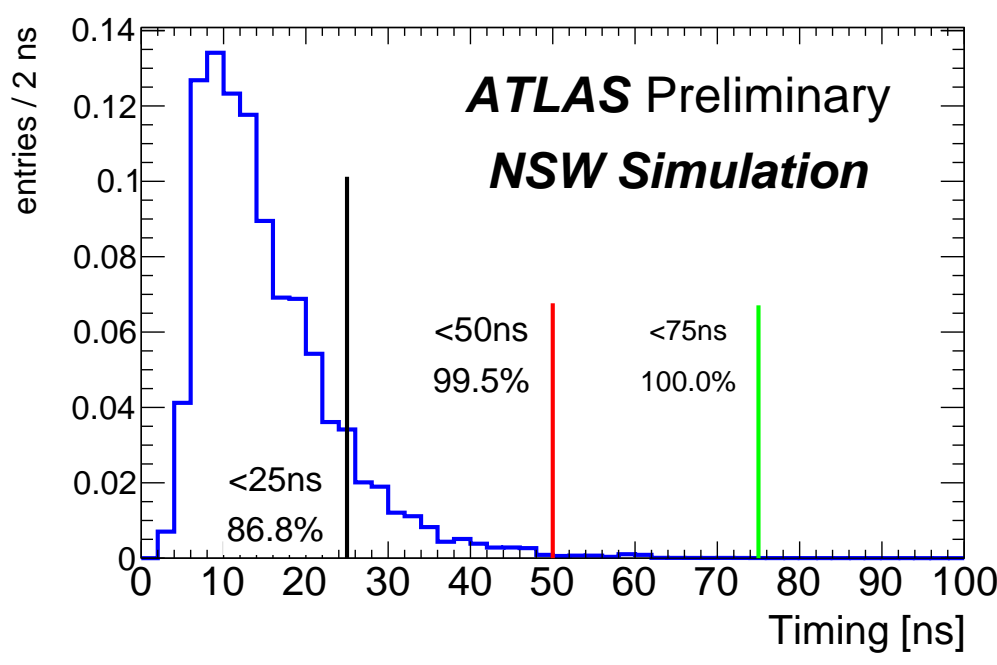

Figure 4: Timing distribution of ART signal. A 25 (50) ns time window has 87\% (99\%) efficiency. The starting point of the time is a hit timing.

\section{NSW MM Trigger algorithms}

The MM trigger processor will find the track-segments and calculate the track parameters. A brief overview of the algorithm implemented into the Trigger Processor (TP) FPGA is described as following [5]:

1. The incoming strip hit addresses (ART signals) are converted into the global position and slope values using a look-up table. The slope value is defined as the orthogonal distance between the given strip and the beam line divided by $z$ coordinate of the detector plane. The hit coincidence of 8-layers is applied to reconstruct a slope-road (see Figure 5a), where each hit has a 50 ns maximum lifetime as discussed in Section 2.

2. The slope-road is used to find a track-segment candidate, by requesting multi-plane hit coincidence in the 8 layers.

- In order to measure the azimuthal angle $\phi$, some MM planes have a slight stereo tilt $\left(1.5^{\circ}\right)$. As shown in Figure 5b, for each MM quadruplet, there are two horizontal strip planes (labeled as $\mathrm{X}$ ) and two stereo strip planes (labeled as $\mathrm{U}$ and $\mathrm{V}$ depending on the direction). 
- The coincidence independently requires a minimum number of $\mathrm{X}$ and $(\mathrm{U}, \mathrm{V})$ planes to be hit in a given slope-road. For example, $3 \mathrm{X} 3 \mathrm{UV}$ ( $\geqslant 3$ hits in $\mathrm{X}$, plus $\geqslant 3$ hits in $\mathrm{U}+\mathrm{V}$ planes). In the simulation studies in Section 4 , three different coincidence thresholds (4X4UV, 3X3UV and 2X2UV) will be performed and compared.

3. After the hit coincidence, the two-dimensional position $(\eta, \phi)$ and $\Delta \theta$ for the track-segment are calculated. The $\eta$ is derived from an average on the slopes of $X$ plane hits, whereas the hits on the slopes of $\mathrm{U}$ and $\mathrm{V}$ plane are converted to the azimuthal angle $\phi$ using a look-up table. The $\Delta \theta$ is defined as an angular deviation of MM track-segment with respect to an infinite momentum track coming from the IP. A $15 \mathrm{mrad}$ cut will be applied on the $\Delta \theta$ in order to eliminate non-IP pointing background.

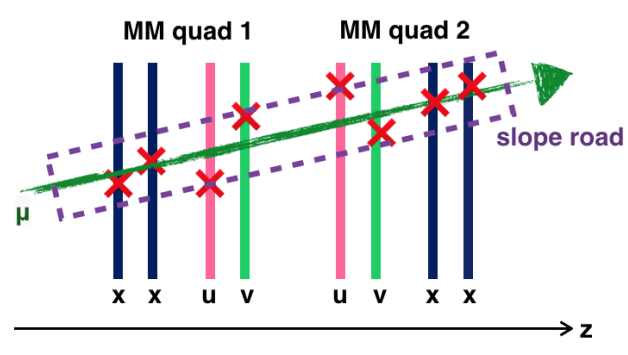

(a) The hit coincidence of $8 \mathrm{MM}$ layers is applied into a slope-road.

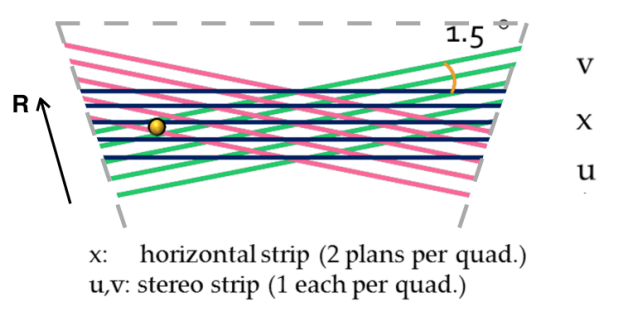

(b) In each MM quadruplet, there will be 2 horizontal strip planes $(\mathrm{X})$ and 2 stereo strip planes $(\mathrm{U}, \mathrm{V})$, where the stereo strip planes have $1.5^{\circ}$ inclined strip angles

\section{Performance of the MM trigger system}

\subsection{Track-segment finding efficieny}

Figure 5a shows track-segment finding efficiencies as a function of the mean number of interactions per bunch crossing $\langle\mu\rangle$, where the hit rate condition at $\langle\mu\rangle=80$ (160) corresponds to the scenario of LHC Run3 (High-Luminosity LHC). The tightest condition 4X4UV gets the lowest efficiency as expected. Also the efficiency of $4 \mathrm{X} 4 \mathrm{UV}$ decreases in particular high $\langle\mu\rangle$, while the 3X3UV and 2X3UV coincidences maintain as high as 98\%. The increase of inefficiencies with respect to large $\langle\mu\rangle$ is well understood as the reasons of front-end electronics deadtime, and the increase of pileup events.

Figure 5b shows the efficiencies of track-segment finding as a function of $\eta$ for various coincidence threshold and different $\langle\mu>$ circumstances. The efficiencies drop a few percentage at high $\eta$ region at $3 \mathrm{X} 3 \mathrm{UV}$ coincidence when $\langle\mu\rangle=80$ and 160 . This is because the extreme increase of the hit rate in high $\eta$ region. Since the $|\eta|$ coverage of NSW trigger is only up to 2.4 , the impact of this drop is negligible.

\subsection{Track-segment rate}

Figure 5 shows the average numbers of track-segments per bunch crossing for various coincidence thresholds. The sample used in the simulation is the minimum bias events. The number of 


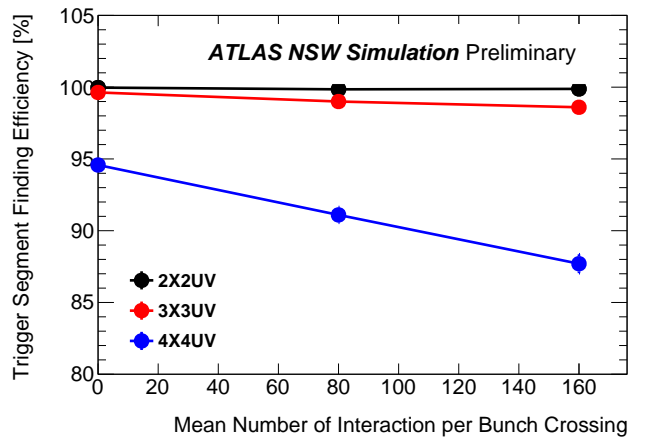

(a) Track-segenment finding efficiencies as a function of mean number of interactions per bunch crossing $<\mu>$, for varies hit coincidence thresholds.

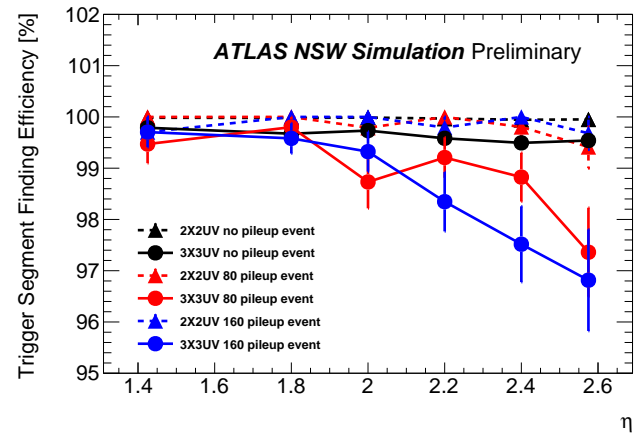

(b) Track-segment finding efficiencies as a function of $\eta$, for varies hit coincidence thresholds and multiple pile-up circumstances. The NSW $|\eta|$ coverage region is up to 2.4 .

track-segments that could be sent to the Sector Logic is limited at most 8 per bunch crossing due to the bandwidth (two optical fibers of $6.4 \mathrm{Gbps}$ ). The 3X3UV coincidence has less than one tracksegment even at $\langle\mu\rangle=160$, whereas the $2 \mathrm{X} 2 \mathrm{UV}$ coincidence has about 2.5 track-segments per bunch crossing. It also has been checked that a few events of $2 \mathrm{X} 2 \mathrm{UV}$ coincidence have numbers of track-segments per bunch crossing more than 8 , which exceed the maximum allowed. Therefore the $3 \mathrm{X} 3 \mathrm{UV}$ should be considered as the best coincidence option in terms of track-segment finding efficiency and track-segment rate.

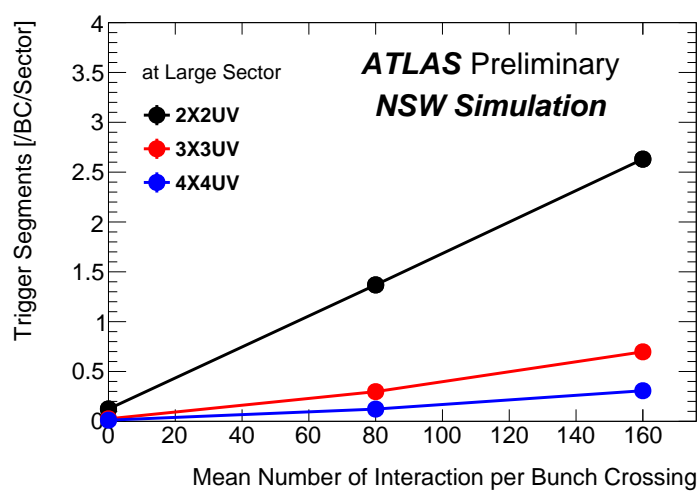

Figure 5: Average number of track-segments per sector, simulated by a minimum bias sample for various hit coincidence thresholds.

\subsection{Resolution of track-segment angular parameters}

Figure 6 shows the residual distributions of the measured value $(\eta, \phi)$ and $\Delta \theta$, demanding the 3X3UV hit coincidence at $\langle\mu\rangle=160$. Resolution from these distributions are $4.7 \times 10^{-5}$ for $\eta$, $1.9 \mathrm{mrad}$ for $\phi$, and $1.1 \mathrm{mrad}$ for $\Delta \theta$. The measured resolutions are all better than the required values, which are $5 \times 10^{-3}$ for $\eta, 20 \mathrm{mrad}$ for $\phi$ and $1.0 \mathrm{mrad}$ for $\Delta \theta$. 


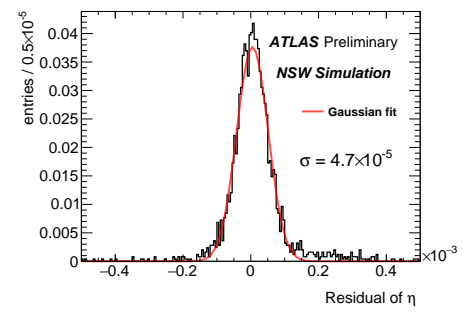

(a) $\eta$ residual

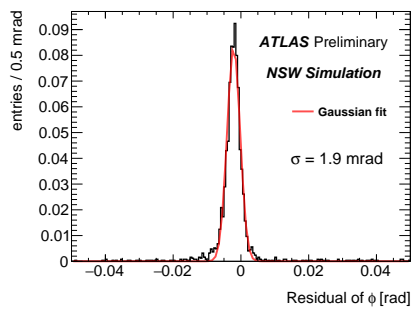

(b) $\phi$ residual

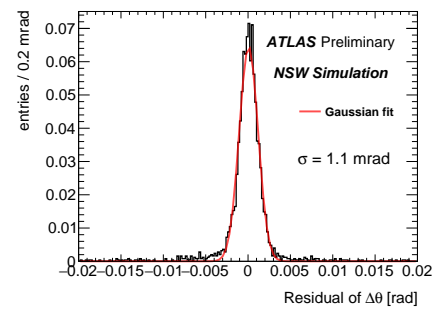

(c) $\Delta \theta$ residual

Figure 6: Residual of measured $\eta, \phi$ and $\Delta \theta$ with respect to the truth tracks, obtained by tracking of GEANT4 hits, at the 3X3UV hit coincidence threshold and $\langle\mu\rangle=160$.

\section{Conclusions}

The ATLAS NSW is motivated to improve the tracking efficiency and reduce the fake trigger rate in future LHC runs. Two new technologies (MM and sTGC) are employed for tracking and triggering independently. The designed electronics for both MM and sTGC has been almost completed. The trigger algorithms have been developed and currently being commissioned in hardware. The simulations show the good performance at efficiencies and resolutions. In the simulation, different numbers of interactions per bunch crossing have been considered. The results of simulation show that the track-segment finding efficiency is as high as $98 \%$, and the average track-segment rate per bunch crossing is much smaller than the maximum limit 8 . In the meanwhile, the resolutions of the angular parameter all satisfy the design requirements, even at the highest pile-up condition. The results of performed studies demonstrate that the MM trigger systems is capable for working with good muon reconstruction efficiencies.

\section{References}

[1] ATLAS Collaboration, The ATLAS Experiment at the CERN Large Hadron Collider, JINST 3 S08003 (2008).

[2] The NSW Trigger Processor Working Group, Trigger Processor Design Review Report (2015).

[3] ATLAS New Small Wheel Collaboration, New Small Wheel Technical Design Report, ATLAS-TDR-020 (2013).

[4] ATLAS Colloboration, Technical Design Report for the Phase-I Upgrade of the ATLAS TDAQ System, ATLAS-TDR-023 (2013).

[5] B. Clark, et al., An Algorithm for Micromegas Segment Reconstruction in the Level-1 Trigger of the New Small Wheel, ATL-COM-UPGRADE-2014-012 (2014) 